Sedó, P. (2020). Reencuentro con el trueque y los sabores familiares en el ámbito comunitario de Costa

Rica, en medio de la pandemia COVID-19. Revista Herencia 33 (2), julio-diciembre, 103-118.

\title{
REENCUENTRO CON EL TRUEQUE Y LOS SABORES FAMILIARES EN EL ÁMBITO COMUNITARIO DE COSTA RICA, EN MEDIO DE LA PANDEMIA COVID-19
}

Meet the barter and the family flavors in the community field of Costa Rica, in the midst of the COVID-19 pandemic

\author{
Patricia Sedó Masís \\ Universidad de Costa Rica, Costa Rica \\ patricia.sedo@ucr.ac.cr
}

Recibido: 30-07-2020

Aprobado: 05-08-2020

\begin{abstract}
Patricia Sedó Masís es docente e investigadora de la Escuela de Nutrición de la Universidad de Costa Rica, desde 1989, en temas sobre cultura alimentaria y nutrición geriátrica. Posee Maestría en Gerontología, Maestría en Administración Educativa y Licenciatura en Nutrición, miembro de la Cátedra de Patrimonio y Diversidad Cultural de la UCR y de la Asociación de Alimentos y Cocinas Regionales de América.
\end{abstract}

\section{RESUMEN}

Se describen aspectos socioculturales, de producción, sociales familiares y comunitarios asociados con la alimentación en el contexto de la pandemia de COVID-19, a partir de una revisión documental y 20 entrevistas semiestructuradas realizadas entre abril y julio del 2020 a líderes comunitarios y productores de diversas partes de Costa Rica (11 mujeres y 9 varones con un promedio de edad de 47,5 años). El distanciamiento físico, mayor permanencia en casa, pérdida del poder adquisitivo familiar, cambios en las dinámicas laborales, asociación colaborativa de productores locales, ayuda humanitaria e incremento de iniciativas productivas y emprendimientos familiares para generar entradas económicas, y sobrevivir en medio de la pandemia, son altamente relevantes en el contexto actual de inseguridad alimentaria y nutricional. Se evidencia la necesidad de retomar la cocina como un espacio de encuentro familiar, y el seguimiento de recetas familiares. Se presenta la revalorización de los alimentos producidos nacionalmente e interés por su trazabilidad, así como prácticas de recolección de alimentos subutilizados y la producción para autoconsumo. Resalta también la toma de medidas para evitar la pérdida y el desperdicio de alimentos en un contexto de crisis económica y ahorro familiar.

Palabras clave: Costa Rica; alimentación; COVID-19; cultura; cultura alimentaria

\section{ABSTRACT}

Sociocultural, production, social family and community aspects associated with food are described in the context of the COVID-19 pandemic, based on a documentary review and 20 semi-structured interviews conducted between April and July 2020 with community leaders and producers of various parts of Costa Rica (11 women and 9 men with an average age of 47.5 years). Physical distancing, greater stay at home, loss of family purchasing power, changes in labor dynamics, collaborative association of local producers, humanitarian aid and increased productive initiatives and family ventures to generate economic income, and survive in the midst of the pandemic, they are highly relevant in the current context of food and nutritional insecurity. The need of people to retake the kitchen as a family meeting space, and the 
monitoring of family recipes is evident. The revaluation of nationally produced food and interest in its traceability are presented, as well as practices for collecting underused food and production for selfconsumption. It also highlights the taking of measures to prevent food loss and waste in a context of economic crisis and family savings.

Keywords: Costa Rica; food; COVID-19; culture; food culture

\section{Introducción}

La emergencia sanitaria en Costa Rica se declaró según Decreto Ejecutivo 42227-MP-S, publicado el 16 de marzo del 2020. A finales de julio, la COVID-19 afectaba cerca de 16 millones de personas en el mundo, y en Costa Rica la cifra se acerca a los 20.000 casos y 150 muertes (Coronavirus Research Center, 2020).

Conforme avanza el tiempo se agudizan los problemas sanitarios, sociales y económicos que afectan la dimensión individual y colectiva para satisfacer las necesidades primarias, entre ellas la alimentación (CEPAL/FAO, 2020a).

En comparación con el año 2019, la tasa nacional de desempleo, medida entre marzo-abril 2020, alcanza el 20,1\%, con una mayor afectación en mujeres (26\%) en comparación con varones (16,3\%). La tasa de subempleo está en un $17,6 \%$, y el porcentaje de personas económicamente activas que se encuentran fuera de la fuerza laboral es del $41,6 \%$, siendo las mujeres las mayormente afectadas (54\%) (INEC,2020).

La reducción en los ingresos económicos a nivel familiar y comunitario está provocando inseguridad alimentaria y nutricional, lo cual es notorio en la mayoría de países, y Costa Rica no es la excepción (FAO, 2020; CEPAL/FAO, 2020a).

La estrategia llamada "la danza y el martillo", propuesta por Tomás Pueyo, y asumida por el gobierno costarricense en el esfuerzo por controlar la situación sanitaria nacional, se basa en un equilibrio entre medidas restrictivas y de flexibilización en las actividades sociales, productivas y comerciales, según la situación epidemiológica (Pueyo, 2020; Ministerio de Salud [MS], 2020). Las medidas se aplican según la situación epidemiológica que enfrentan las diferentes regiones del país (MS, 2020). 
Existen variaciones en los niveles de desarrollo socio económico y otras condiciones que provocan diferencias intercantonales, en el mismo cantón, distritos y barriadas del país. Ello es de gran preocupación para las personas entrevistadas, quienes refieren un trato sin equidad, y cierto grado de desconocimiento y descoordinación por parte de las instancias nacionales y locales en la aplicación de las medidas restrictivas.

Paralelamente se señala la falta de diálogo entre el gobierno y los diferentes sectores, siendo las organizaciones sociales y representantes de la Cámara Nacional de la Economía Social Solidaria los que demandan mayor equidad en las mesas de negociación (Chacón, 2020; Cordero, 2020).

Ante el incremento del contagio, la reactivación económica es incierta para muchos sectores. Aquellas poblaciones dependientes totalmente de las actividades agroproductivas, pesca, turismo y gastronomía presentan muy pocas o nulas oportunidades para su mantenimiento o reactivación, con el consecuente incremento del subempleo y desempleo. Ello conduce a la mayor pobreza y subdesarrollo (CEPAL, 2020; OCEX, 2020).

El turismo internacional está paralizado, mientras que el nacional ha mostrado inactividad en los últimos meses (Estado de la Nación, 2020). La Cámara Costarricense de Restaurantes y Afines refiere, según datos de abril del 2020, que cerca del $45 \%$ de negocios afiliados dejó operaciones, lo cual representa 8.550 locales y un $53 \%$ de trabajadores despedidos (CACORE, 2020).

La reactivación económica tomará su tiempo, mientras las personas dependientes de diversas actividades productivas miran con desesperanza e incertidumbre el futuro inmediato, principalmente en cantones deprimidos económicamente, zonas costeras, fronterizas y urbano marginales.

El presente estudio describe aspectos socioculturales, de producción, sociales familiares y comunitarios asociados con la alimentación en el contexto de la pandemia por COVID-19 en Costa Rica.

\section{Método de trabajo}

La investigación es cualitativa, y forma parte del proyecto EC-436 "Tradiciones alimentarias y cocina patrimonial de Costa Rica: aportes para contribuir con su salvaguardia" de la Escuela de Nutrición de la Universidad de Costa Rica. 
Comprende una revisión documental y 20 entrevistas semiestructuradas por teléfono o videollamadas. Las mismas se aplicaron entre abril y julio del 2020 a líderes comunitarios, productores y emprendedores de diversas partes del país.

La participación fue voluntaria, 11 mujeres y 9 varones con un promedio de edad de 47,5 años. Se brindó información sobre objetivos, alcances e implicaciones de participación. Se realizó la transcripción de la información y el ordenamiento y análisis de la misma en categorías, según objetivo establecido.

\section{Cambios en la alimentación y seguridad alimentaria y nutricional en la población costarricense}

De acuerdo con estudios en América Latina y el Caribe, la pandemia de COVID19 ha contribuido a una mayor vulnerabilidad alimentaria, tanto en suficiencia de alimentos como en calidad nutricional de la dieta (FAO/CELAC, 2020; CEPAL/FAO, 2020a, 2020b).

A propósito de lo anterior, una persona dedicada a la pesca artesanal en Puntarenas mencionó: "La gente acá está literalmente muriendo de hambre. No hay trabajo, no hay comida, no hay qué hacer, y eso no es de ahora (...) desde antes de la llegada del virus, estabamos mal, y ahora peor" (E4).

Ante la falta de empleo y reducción de las finanzas familiares, muchas personas han optado por actividades informales, ocasionales o con relativa permanencia en el tiempo. También se ha incrementado la demanda de ayuda alimentaria entre vecinos, organizaciones e instituciones locales, con lo cual los bancos de alimentos constituyen una respuesta paliativa ante la crisis humanitaria (FAO, 2020).

Las personas entrevistadas perciben que cada vez son más las familias en sus comunidades que han modificado su alimentación, señalando la menor adquisición de productos de alto costo, como carne, lácteos u otros productos. Se presenta la reducción en cantidades de alimentos servidos u omisión en tiempos de comida; la privación de alimentos en algunos miembros para alimentar a las personas más vulnerables y/o recurrir a la ayuda alimentaria fuera de sus familias o conocidos, ante la insuficiencia.

En el Cuadro 1 se resumen varios aspectos asociados con lo señalado anteriormente. 
Revista Herencia, Vol. 33 (2), julio-diciembre, 2020.

Cuadro 1. Descripción de aspectos asociados con la seguridad alimentaria y nutricional de la población costarricense en el contexto de la pandemia de COVID-19.

\begin{tabular}{|c|c|c|c|}
\hline Ámbito & Adversidades & Implicaciones & Acciones, oportunidades y retos \\
\hline $\begin{array}{c}\text { Nacional y } \\
\text { comunitario }\end{array}$ & $\begin{array}{l}\text { Aumento de familias } \\
\text { en condición de } \\
\text { pobreza. } \\
\text { Grandes pérdidas } \\
\text { para el sector agro } \\
\text { exportador, turismo } \\
\text { y gastronomía. } \\
\text { Afectación seria a } \\
\text { pequeños negocios } \\
\text { Cierre de los } \\
\text { servicios de } \\
\text { comedor de } \\
\text { programas } \\
\text { nacionales. } \\
\text { Adopción del } \\
\text { trabajo remoto en } \\
\text { gran parte de } \\
\text { instituciones del } \\
\text { sector público y } \\
\text { privado. } \\
\text { Aumento del } \\
\text { subempleo y } \\
\text { desempleo. } \\
\text { Hacinamiento social } \\
\text { y riesgo de contagio. }\end{array}$ & $\begin{array}{l}\text { Subalimentación y } \\
\text { hambre. } \\
\text { Alteraciones en los } \\
\text { sistemas alimentarios } \\
\text { locales. } \\
\text { Cambios en la } \\
\text { provisión alimentaria en } \\
\text { el ámbito laboral y en } \\
\text { instituciones. } \\
\text { Pérdidas y } \\
\text { endeudamiento en } \\
\text { diferentes sectores. } \\
\text { Incremento de trabajo } \\
\text { informal y ventas } \\
\text { ambulantes. } \\
\text { Cambios temporales en } \\
\text { modos de vida para } \\
\text { reducir riesgos de } \\
\text { contagio. } \\
\text { Desintegración familiar, } \\
\text { al tener personas } \\
\text { infectadas que alejarse } \\
\text { del núcleo y vivir en } \\
\text { albergues para reducir } \\
\text { el contagio. }\end{array}$ & 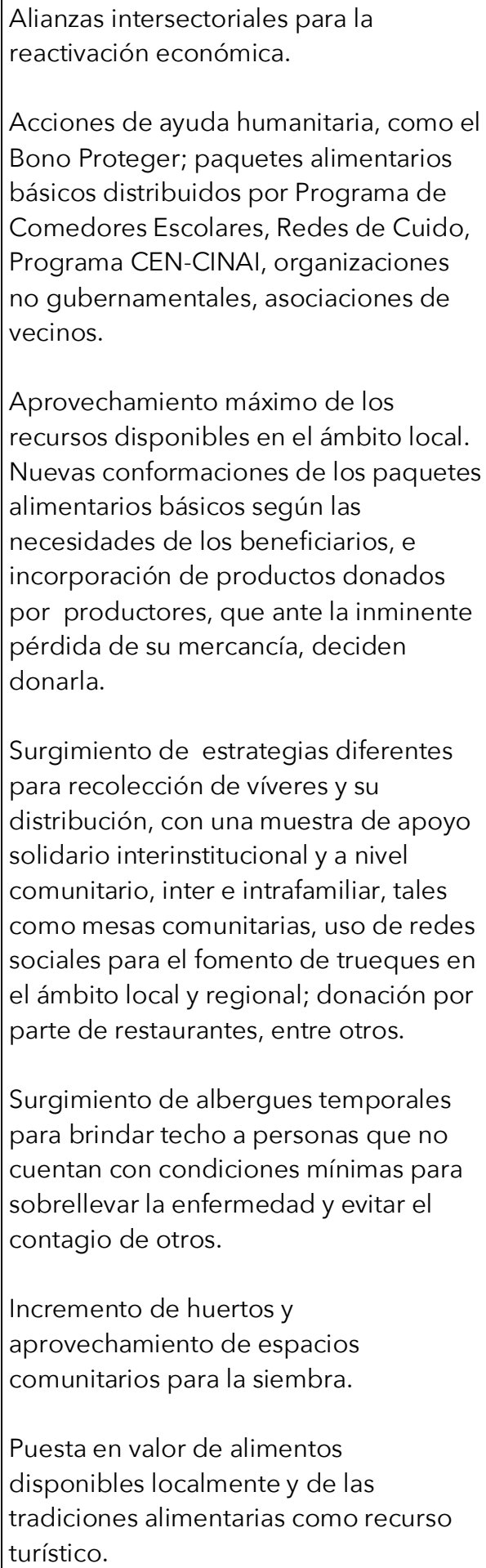 \\
\hline
\end{tabular}


Revista Herencia, Vol. 33 (2), julio-diciembre, 2020.

cont. Cuadro 1.

\begin{tabular}{|c|c|c|c|}
\hline Ámbito & Adversidades & Implicaciones & $\begin{array}{c}\text { Acciones, oportunidades y } \\
\text { retos }\end{array}$ \\
\hline Familiar & $\begin{array}{l}\text { Cambios en las formas de } \\
\text { abastecimiento y preparación } \\
\text { de alimentos. } \\
\text { Riesgos por inocuidad } \\
\text { alimentaria. } \\
\text { Incremento en el gasto de } \\
\text { servicios básicos de agua y } \\
\text { energía eléctrica, así como del } \\
\text { servicio de red internet en el } \\
\text { ámbito domiciliar. }\end{array}$ & $\begin{array}{l}\text { Pérdida del poder } \\
\text { adquisitivo de } \\
\text { alimentos. } \\
\text { Modificación en los } \\
\text { patrones alimentarios } \\
\text { en las familias, y a nivel } \\
\text { intrafamiliar. } \\
\text { Surgimiento de nuevas } \\
\text { prácticas de compra y } \\
\text { preparación de } \\
\text { alimentos y } \\
\text { reforzamiento o cambio } \\
\text { de roles intra familiares } \\
\text { para solventar las } \\
\text { necesidades. } \\
\text { Las alteraciones } \\
\text { dietéticas pueden } \\
\text { afectar el bienestar y } \\
\text { salud de la familia. } \\
\text { Reforzamiento de } \\
\text { buenas prácticas de } \\
\text { higiene y manipulación } \\
\text { de alimentos. }\end{array}$ & $\begin{array}{l}\text { Prácticas de ahorro en } \\
\text { alimentos y reducción de } \\
\text { pérdidas y desperdicio. } \\
\text { Mayor interés por la } \\
\text { autosuficiencia alimentaria e } \\
\text { incremento de huertas } \\
\text { caseras, lo cual contribuye a la } \\
\text { diversificación de la dieta. } \\
\\
\text { Oportunidad de adquisición } \\
\text { de productos locales que en } \\
\text { otros momentos eran } \\
\text { inaccesibles mediante trueque } \\
\text { o por compra directa a } \\
\text { productores locales o } \\
\text { nacionales. Resalta también el } \\
\text { uso de productos comestibles } \\
\text { obtenidos por recolección del } \\
\text { medio. } \\
\text { Necesidad de preparar la } \\
\text { propia comida, con una } \\
\text { revalorización de las recetas } \\
\text { familiares y la comensalidad. }\end{array}$ \\
\hline $\begin{array}{c}\text { Sector } \\
\text { alimentario }\end{array}$ & $\begin{array}{l}\text { Cierres temporales o } \\
\text { permanentes de los mercados } \\
\text { y negocios por medidas } \\
\text { sanitarias, lo que provoca } \\
\text { insostenibilidad económica. }\end{array}$ & $\begin{array}{l}\text { Reforzamiento de } \\
\text { buenas prácticas de } \\
\text { higiene y manipulación } \\
\text { de alimentos. } \\
\text { Urgente reinvención de } \\
\text { formas de } \\
\text { comercialización de } \\
\text { alimentos para } \\
\text { mantener la } \\
\text { sostenibilidad, con } \\
\text { incursión al mercadeo } \\
\text { digital. } \\
\text { Reinvención de los } \\
\text { servicios gastronómicos } \\
\text { con cambios en la } \\
\text { oferta y formas de } \\
\text { distribución para poder } \\
\text { llegar a los clientes. }\end{array}$ & $\begin{array}{l}\text { Necesidad de mayor } \\
\text { conocimiento y manejo de } \\
\text { herramientas digitales para el } \\
\text { comercio y mercadeo. } \\
\text { Interés de los consumidores } \\
\text { por la producción nacional, y } \\
\text { cercanía al productory } \\
\text { comerciante detallista. } \\
\text { Interés del consumidor por la } \\
\text { trazabilidad de alimentos. } \\
\text { Desarrollo de nuevas } \\
\text { capacidades por parte de } \\
\text { productores y distribuidores } \\
\text { para favorecer } \\
\text { encadenamientos y reducir } \\
\text { pérdidas de productos. }\end{array}$ \\
\hline
\end{tabular}


cont. Cuadro 1.

\begin{tabular}{|c|c|c|c|}
\hline $\begin{array}{c}\text { Ámbito } \\
\text { alimector }\end{array}$ & Adversidades & $\begin{array}{l}\text { Implicaciones } \\
\text { retos }\end{array}$ & $\begin{array}{l}\text { Acciones, oportunidades y } \\
\text { retos }\end{array}$ \\
\hline $\begin{array}{l}\text { Proliferación de } \\
\text { emprendimientos } \\
\text { familiares para la venta } \\
\text { de productos } \\
\text { procesados } \\
\text { artesanalmente y } \\
\text { comidas preparadas. }\end{array}$ & $\begin{array}{l}\text { Fortalecimiento del trueque } \\
\text { entre productores, } \\
\text { restaurantes y } \\
\text { emprendimientos para una } \\
\text { mayor diversificación de la } \\
\text { oferta, y solventar necesidades } \\
\text { de producción. }\end{array}$ \\
$\begin{array}{l}\text { Conformación de redes para la } \\
\text { promoción y venta de } \\
\text { alimentos y comidas } \\
\text { preparadas a partir de } \\
\text { asociaciones locales, apoyo de } \\
\text { municipalidades, iglesias u } \\
\text { otras instancias. }\end{array}$ \\
$\begin{array}{l}\text { Innovación en servicios más } \\
\text { allá de la oferta de productos } \\
\text { alimenticios, con valores } \\
\text { agregados, como empaques } \\
\text { artesanales y/o amigables con } \\
\text { el ambiente, elaboración de } \\
\text { canastas de productos } \\
\text { preenvasados o ingredientes } \\
\text { para la elaboración de } \\
\text { preparaciones especificas, } \\
\text { entre otros. }\end{array}$ \\
\hline
\end{tabular}

Fuente: Elaboración propia, julio 2020.

Se señala la necesidad de buscar mayor unión intersectorial e interinstitucional, con acciones oportunas y pertinentes que lleguen a todas las regiones del país, principalmente a las más afectadas.

Es innegable las pérdidas que enfrentan los agricultores debido al cierre de las operaciones turísticas, exportación, merma en los negocios gastronómicos y el cierre de comedores institucionales. 
En las ferias del agricultor se presenta una disminución en ventas debido a la menor afluencia de compradores. Al respecto, un agricultor que participa en la Feria del Agricultor de Desamparados indicó lo siguiente:

\footnotetext{
"Diay, con esto del COVID uno no sabe ni qué hacer. Me fui el sábado temprano, puesto que la noticia desde la Municipalidad era que la feria estaría abierta. Llego en la madrugada y un policía me dice que no, que según la directriz del Ministerio está cerrada. Me quedé con la mercadería. Como a las dos horas esperé si ninguna solución, y luego me tuve que ir hacia la casa (...) y preocupado, pensando si la feria se mantendrá abierta o no, y me pregunto ¿qué voy a hacer si la cosa sigue así?, con deudas y una familia que darle de comer." (E19).
}

Resalta la diversificación de acciones ante la crisis, como la asociación con otros productores que asisten a diferentes ferias, el contacto con otros puntos de venta o de forma directa con los consumidores, lo cual genera para algunos mayor esfuerzo, estrés y gasto.

También se recurre al apoyo en cooperativas y cámaras, organizaciones e instituciones locales, donde algunas municipalidades han asumido un liderazgo importante; sin embargo se menciona la necesidad de una mayor coordinación entre los gobiernos locales y el central.

De igual forma sobresale la incapacidad de productores la falta de tecnología y otros recursos para el almacenamiento y conservación de sus productos. La fluidez de caja les impide a muchos incursionar en nuevas formas de comercialización, con el agravante de pérdidas importantes, incluyendo el desecho de grandes cantidades de alimento por no disponer de mercados o poder comercializarlo a tiempo.

En algunos casos se ha enfrentado el abandono de los terrenos cultivados, lo cual genera gran tristeza y frustración, dados los meses de trabajo y recursos invertidos; al tiempo que se piensa en un futuro prometedor que se resquebrajó de manera abrupta con la llegada del coronavirus, tal como lo describió un productor de la zona norte del país.

Los negocios gastronómicos que tuvieron que cerrar sus puertas para la atención al público se vieron presionados a reinventarse para sobrevivir, aunque muchos cerraron operaciones. Es notoria la diversificación en la oferta gastronómica y el fortalecimiento del servicio a domicilio o entrega en puerta del negocio. Se presenta un trabajo asociativo entre personas de los sectores 
agropecuario, gastronómico y artesanía para el desarrollo de nuevas iniciativas, tales como la venta de canastas de alimentos preenvasados, preparados o semi elaborados, nuevos empaques y elementos identitarios que agregan valor a los productos locales, y la oferta de nuevos menús.

También sobresalen los emprendimientos dedicados a comidas preparadas por parte de personas que han quedado desempleadas, quienes anteriormente no estaban involucradas con el sector alimentario, dedicadas a la venta de tamales, panes, repostería, cajetas, productos envasados (jaleas, encurtidos, salsas y condimentos naturales), picadillos, tortillas, empanadas, chorreadas, bizcocho, comidas rápidas y otros.

La amplia oferta gastronómica va desde restaurantes especializados a pequeños emprendimientos familiares, lo cual contrasta con la reducción de ingresos económicos de los consumidores e incapacidad de compra.

Las personas dedicadas a ferias comunitarias, turnos y otros espacios, cuyos fondos son dedicados principalmente a obras sociales, muestran tristeza y preocupación por el cierre. Más allá del aspecto económico, resalta la pérdida estos espacios comunitarios, tan necesarios para la socialización y difusión de las tradiciones locales.

Al respecto una persona entrevistada manifestó:

\footnotetext{
"Pues acá es altamente triste ver cómo no pudimos realizar la feria, que siempre trae bendiciones para los pequeños productores y artesanos cada año. Es un espacio para compartir, para unirnos como pueblo, ahora cada uno está en su casa, y con miedo." (E1).
}

En medio de la crisis, surgen nuevas formas de organización. Ante la imposibilidad de celebrar el turno patronal o ferias, en algunas parroquias han puesto a disposición la venta de comidas haciendo uso de redes sociales. Algunas iniciativas son: la "Ventanita de San Ignacio" (San Ignacio de Acosta); el "Fogón de Fátima" (Hatillo 3); el "Ranchito de Moncho" (Sabanilla Montes de Oca); la "Cocina Parroquial" (Santa Ana centro); el "Turno express" (Paraíso de Cartago), y la venta de pan casero y repostería en la Parroquia de Pocosol en San Carlos, o chorreadas y vainicas producidas en el terreno parroquial (Cuatro Reinas de Tibás). 
Como principales barreras encontradas están la falta de acceso a nuevas tecnologías y recursos para incursionar en el mercadeo digital y complejidad logística para ofrecer o acceder a los nuevos servicios, así como la limitación geográfica para la distribución.

\section{El reencuentro con el trueque}

El trueque es una forma de intercambio comercial común desde la antigüedad, el cual repunta con la pandemia. Sin que medie dinero, y a partir de negociaciones consensuadas, las personas logran satisfacer algunas necesidades básicas.

Se fundamenta en la economía social solidaria y la necesidad de apoyo mutuo entre personas que residen en una misma localidad, aunque es interesante el intercambio entre zonas alejadas, tal como sucede con productores de hortalizas de Cartago y pescadores de Puntarenas.

De igual forma, resalta el "Estanco indígena de trueque virtual productivo" promovido conjuntamente por la Asociación de Mujeres Kábata Könana, la Asociación de Desarrollo Integral del Territorio Indígena Talamanca Cabécar y la Red Indígena Bribri y Cabécar, con el fin de abastecer de productos comestibles, semillas y otros insumos a las comunidades indígenas bribri y cabécar, aunque es importante señalar que el trueque es una actividad natural que se gesta en estas comunidades desde siempre.

Los bancos de alimentos se han visto favorecidos con el trueque, dado el acopio y distribución de productos comestibles y otros productos básicos. En la mayoría de los casos destaca el uso de redes sociales para facilitar el intercambio de bienes y servicios en el ámbito local.

El favorecimiento de la economía local también se presenta con la adquisición de productos básicos por parte de la Comisión Nacional de Prevención de Riesgos y Atención de Emergencias y otras instancias públicas para la distribución de paquetes de alimentos a familias afectadas por la crisis. 


\section{El reecuentro con la tierra y el alimento}

En tiempos de crisis alimentaria, resulta imprescindible recurrir a la obtención de productos del medio, así como el fortalecimiento de la producción alimentaria para autoconsumo, y la asociación comunitaria para el abastecimiento. Se identificó el mayor interés de las personas por la siembra de hortalizas y cuidado de árboles frutales y animales, como cerdos y gallinas, lo cual contribuye con una mayor diversificación de la dieta.

Hay mayor uso de alimentos subutilizados, a lo que González (2008) denomina "alimentos olvidados". Con la mayor permanencia de las personas en sus casas y en sus comunidades se ha visto la necesidad de recolectar alimentos, tales como quelites, flores, tallos, semillas, hojas como el chicasquil.

Al respecto mencionó un entrevistado:

"La gente no valora lo que tiene hasta que se enfrenta a una gran necesidad. Mis papás eran sumamente pobres, y nunca faltó comida en la casa, porque eran demasiado creativos para sacar provecho de lo que encontraban a su alrededor. Nos hemos acostumbrado a comprar todo y a desvalorizar lo nuestro, pero en crisis, hay que volver los ojos de nuevo a lo que se tiene y dejamos de comer (...) un guineo con frijoles es bien recibido, máxime si es posible arrimarle un huevo y un poquito de arroz." (E7).

Otra informante mencionó:

"Hoy mi papá me trajo un racimo de plátanos que encontró en el camino y un poco de frijoles sembrados por él, y yo le estoy eternamente agradecida. Es una bendición en estos momentos contar con alguito que darles de comer a mis hijos, cuando mi esposo y yo no tenemos trabajo." (E11).

La reutilización de los sobrantes de comida y el aprovechamiento de la mayor parte o la totalidad del alimento forman parte de las acciones mencionadas para reducir los gastos.

\section{El reencuentro con la cocina familiar}

El confinamiento en casa ha motivado a muchas personas a regresar con energía a un espacio vital: la cocina doméstica. Se trata de recobrar y reinventarse en un espacio tan familiar, pero quizás para muchos desconocido, puesto que en su estilo de vida, antes de la pandemia, era más común comer fuera de casa o que otros prepararan su propia comida. El intercambio de información culinaria se suscita a lo interno y fuera del seno familiar. 
Al respecto, Ross (2009) señala que el bombardeo de información alimentaria en diferentes medios, pero particularmente en redes sociales, incide directamente en las conductas alimentarias, y las valoraciones que las personas hacen de la comida, con contraposiciones de lo que se reconoce como "comida tradicional" y el simbolismo de la "fast food", que lleva a la homogeneización de paladares, la pérdida de elementos identitarios de las ricas cocinas locales, y el seguimiento de prácticas comerciales globales aculturizantes.

Lo vivido en los últimos meses, producto de la pandemia, es una importante oportunidad de las personas para reencontrarse con la herencia culinaria, vivirla y compartirla con las nuevas generaciones. Como parte del reencuentro con los sabores familiares, muchas personas se han visto presionadas por aprender y/o volver a cocinar, asumir roles no comunes en su estilo de vida previo al confinamiento, como la compra o preparación, y aprender a disfrutar de la comida, solos o acompañados.

Al respecto mencionó de las señoras entrevistadas:

\footnotetext{
"En el paquete de comida que me dieron en la iglesia venía un paquete de masa, y comencé a hacer tortillas. Había perdido la práctica, pero lo que bien se aprende en la infancia, no se pierde. Y recordé lo que decía mamá de que la tortilla es el mejor alimento." (E17).
}

Las mujeres que conservan un legado culinario que es una fortaleza que les ayuda a enfrentar de mejor manera la crisis, y apoyar en las finanzas familiares, y ante el problema de mayor desempleo en población femenina.

Así, por ejemplo, una señora de Pérez Zeledón caracterizada por su buena cuchara, vio la oportunidad de vender gallinas achotadas. Constituye una comida de sabores familiares propios de la cocina festiva tradicional que muchos anhelan volver a disfrutar, y la demanda fue tal que tuvo que solicitar ayuda a vecinas para poder entregar los crecientes pedidos en la zona.

Por su parte, una vecina de Desamparados vio la oportunidad de hacer tamales, contando con el apoyo de familiares para la venta por redes sociales, y su distribución en distintas partes del Gran Area Metropolitana; igual sucede con personas dedicadas la venta de panes, queques, licores artesanales, chocolatería, salsas y otros con recetas familiares. 
La pérdida de la práctica de cocinar, herencia ancestral que nos hizo humanos, es una tendencia mundial que muy probablemente se transformó con las nuevas dinámicas sociales generadas por la pandemia. Valorar la comida en momentos de apremio económico, es una ganancia. No siempre las personas se percatan de la importancia de los alimentos accesibles, por lo que es la oportunidad de volver la mirada a lo más simple, a lo comestible que está en la inmediatez del hogar y la comunidad.

Aunado a lo anterior, ante la mayor sensibilización de los consumidores hacia los alimentos locales, existe la oportunidad de comprar alimentos frescos directamente al productor, lo cual es de gran valor en cuanto a la identidad culinaria, y la mayor conciencia de lo que se está comiendo.

Por otro lado, en el sector gastronómico se trata de buscar sabores cada vez más auténticos, con el fortalecimiento de una cocina que se aleja de lo estandarizado, foráneo y/o artificial, para dar paso a sabores más naturales y combinaciones inimaginables, con preparaciones caracterizadas por su frescura y explosión en el paladar, tal como lo refiere Ross (2009).

Comidas y bebidas que nos pueden llegar a la casa, en un esfuerzo de movilización comercial, y que con ello estamos ayudando a la economía nacional y a personas luchadoras que trabajan con fuerza y perseverancia, y disponen de una gran capacidad de resiliencia.

De esta forma, se cumple la calificación de los restaurantes por Ross (2009) como eficientes fábricas de comida con variados menús, sin caer en la maquila o estandarización de sabores, y que en Costa Rica se fortalece como resultado de la reactivación del sector.

\section{Ollas comunes, alimentación teñida de solidaridad}

La pobreza y hambre lleva a las personas a unir esfuerzos para solventar sus necesidades básicas. Así, encontramos casos donde el acopio de verduras y una gran cuota de trabajo solidario dan como resultado grandes ollas de alimento, que de forma individual y con las limitaciones presentes no habría sido imposible preparar para alimentar a decenas de familias. 
Olla de carne, sopa de gallina o de albóndigas, con verduras recolectadas en los negocios y en los predios; plátanos, yuca, papas, chayotes combinados con ingredientes presentes en el paquete de alimentos donados a los núcleos familiares.

La sazón comunitaria se basa en el interés común de alimentar a más bocas uniendo esfuerzos para solventar la crisis alimentaria que se vive por la desacelaración económica en diversas zonas del país.

\section{Conclusiones}

Es probable que la inseguridad alimentaria y nutricional se ha agravado en personas que desde antes de la pandemia se encontraban en condición de pobreza; con las medidas restrictivas para controlar la misma, se unen familias que se encontraban con una relativa estabilidad económica antes, pero que actualmente presentan un rápido deterioro en su situación económica.

Las consecuencias de la pandemia dependerán del tiempo en que se prolonguen las situaciones adversas, así como de la capacidad de resiliencia de las familias y comunidades, la manera en que suceda la reactivación económica y el fortalecimiento del mercado laboral, así como del apoyo estatal y privado, y la organización local logrará cambios positivos de manera sostenida a lo largo del tiempo.

En este contexto, el trueque repunta y contribuye en el mayor dinamismo de la economía local, lo cual se une con el reencuentro de las personas con los alimentos propios de sus tradiciones alimentarias, la cocina doméstica y el disfrute de la comensalidad.

Vale la pena preguntarse: ¿Los avances y cambios positivos logrados durante la crisis por COVID-19 se mantendrán posterior a la crisis? ¿Se mantendrán los reencuentros positivos con la cocina, la comensalidad y la alimentación tradicional? ¿Qué sucederá con el trueque y nuevos emprendimientos o reinvenciones en los servicios y productos que emergieron en el contexto de la crisis, lograrán mantenerse a lo largo del tiempo?

El tiempo es el que nos dará la respuesta. 


\section{Bibliografía}

CACORE. (2020). Sector de restaurantes claman por apertura de lunes a domingo. Recuperado de https://www.cacore.cr/300429, 29 de abril 2020.

CEPAL/FAO. (2020a). América Latina y el Caribe ante la pandemia del COVID19. Efectos económicos y sociales. Chile: Informe Especial № 1 de Naciones Unidas, 3 de abril 2020. Recuperado de https://repositorio.cepal.org/bitstream/handle/11362/45337/4/S2000264 es.p df

CEPAL/FAO. (2020b). ¿Cómo evitar que la crisis del COVID-19 se transforme en una crisis alimentaria? Acciones urgentes contra el hambre en América Latina y el Caribe. Chile: Informe Especial, 16 de junio 2020. Recuperado de https://www.cepal.org/es/publicaciones/45702-como-evitar-que-la-crisiscovid-19-se-transforme-crisis-alimentaria-acciones

Chacón, V. (28 de julio de 2020). No todos tienen campo en la mesa de Carlos Alvarado. Semanario Universidad. Recuperado de https://semanariouniversidad.com

Cordero, M. (28 de julio de 2020). Menos "baile y martillo" y más atención comunitaria, recomiendan expertos. Semanario Universidad. Recuperado de https://semanariouniversidad.com

Coronavirus Research Center (2020). World Map Coronavirus. United States: John Hopkins University Medicine. Recuperado de: https://coronavirus.jhu.edu/map.html, 23 de mayo 2020.

Estado de la Nación. (20 de marzo de 2020). Turismo en Costa Rica: un afectado más por la pandemia COVID-19. Recuperado de https://estadonacion.or.cr/turismo-en-costa-rica-un-afectado-mas-por-lapandemia-covid-19/

FAO (2020). COVID-19: Nuestras comunidades más hambrientas y vulnerables se enfrentan a "una crisis dentro de una crisis". Roma. Recuperado de https://reliefweb.int/report/world/covid-19-nuestras-comunidades-m-shambrientas-y-vulnerables-se-enfrentan-una-crisis 
FAO/CELAC. (2020). Seguridad alimentaria bajo la pandemia de COVID-19. México: Coordinación Nacional pro témpore CELAC. Recuperado de http://www.fao.org/3/ca8873es/CA8873ES.pdf

Jiménez, S. (28 de julio de 2020). Indígenas recurren al trueque virtual para garantizar alimentos durante la pandemia. Semanario Universidad. Recuperado de https://semanariouniversidad.com

González, R. (2008). De flores, brotes y palmitos: alimentos olvidados. Agronomía Mesoamericana. 32(2): 83-192.

INEC (2020). Encuesta continua de desempleo, trimestre movil marzo-abril 2020. cifras preliminares de principales indicadores de mercado laboral. San José. Recuperado de https://www.inec.cr/sites/default/files/documetos-bibliotecavirtual/coece-mam2020.pdf

Ministerio de Presidencia/Ministerio de Salud de Costa Rica. (2020). Decreto Ejecutivo $42227 . \quad$ Recuperado de https://www.presidencia.go.cr/bicentenario/wpcontent/uploads/2020/03/Decreto-Ejecutivo-42227-Emergencia-Nacional.pdf

Ministerio de Salud. (2020). Situación Nacional COVID-19/Documentación. San José. Recuperado de https://www.ministeriodesalud.go.cr/index.php/centrode-prensa/noticias/741-noticias-2020/1532-lineamientos-nacionales-para-lavigilancia-de-la-infeccion-por-coronavirus-2019-ncov

OCEX. (2020). Impactos del COVID-19 en la economía costarricense y mundial. San José. Recuperado de https://www.uned.ac.cr/ocex/index.php/124boletines-articulos/556-impactos-del-covid-19-en-la-economia-costarricense-ymundial

Pueyo, T.(19 de marzo de 2020). Coronavirus: The hammer and the dance. [Mensaje en un blog]. Recuperado de https://medium.com/@tomaspueyo/coronavirus-the-hammer-and-the-dance$\underline{\text { be9337092b56 }}$

Ross, M. (2009). Los siete pasos de la danza del comer. Cultura, género e identidades. San José: Editorial Universidad de Costa Rica. 